\title{
Ventromedial hypothalamus-specific Ptpn1 deletion exacerbates diet-induced obesity in female mice
}

\author{
Franck Chiappini, ${ }^{1}$ Karyn J. Catalano, ${ }^{1}$ Jennifer Lee, ${ }^{1}$ Odile D. Peroni, ${ }^{1}$ Jacqueline Lynch, ${ }^{1}$ Abha S. Dhaneshwar, ${ }^{1}$ Kerry Wellenstein, ${ }^{1}$ \\ Alexandra Sontheimer, ${ }^{1}$ Benjamin G. Neel, ${ }^{2}$ and Barbara B. Kahn ${ }^{1}$
}

'Division of Endocrinology, Diabetes and Metabolism, Beth Israel Deaconess Medical Center and Harvard Medical School, Boston, Massachusetts, USA. ²Princess Margaret Cancer Center, University Health Network and Department of Medical Biophysics, Toronto, Ontario, Canada.

\begin{abstract}
Protein-tyrosine phosphatase 1B (PTP1B) regulates food intake (FI) and energy expenditure (EE) by inhibiting leptin signaling in the hypothalamus. In peripheral tissues, PTP1B regulates insulin signaling, but its effects on CNS insulin action are largely unknown. Mice harboring a whole-brain deletion of the gene encoding PTP1B (Ptpn1) are lean, leptinhypersensitive, and resistant to high fat diet-induced (HFD-induced) obesity. Arcuate proopiomelanocortin (POMC) neuronspecific deletion of Ptpn1 causes a similar, but much milder, phenotype, suggesting that PTP1B also acts in other neurons to regulate metabolism. Steroidogenic factor-1-expressing (SF-1-expressing) neurons in the ventromedial hypothalamus (VMH) play an important role in regulating body weight, FI, and EE. Surprisingly, Ptpn1 deletion in SF-1 neurons caused an age-dependent increase in adiposity in HFD-fed female mice. Although leptin sensitivity was increased and FI was reduced in these mice, they had impaired sympathetic output and decreased EE. Immunohistochemical analysis showed enhanced leptin and insulin signaling in VMH neurons from mice lacking PTP1B in SF-1 neurons. Thus, in the VMH, leptin negatively regulates $\mathrm{FI}$, promoting weight loss, whereas insulin suppresses $\mathrm{EE}$, leading to weight gain. Our results establish a novel role for PTP1B in regulating insulin action in the VMH and suggest that increased insulin responsiveness in SF-1 neurons can overcome leptin hypersensitivity and enhance adiposity.
\end{abstract}

\section{Introduction}

Obesity has reached epidemic proportions in the US and other developed and developing nations. Due to the numerous complications associated with obesity, obesity-related healthcare costs consume $\$ 147$ billion annually, approximately $9 \%$ of total healthcare expenditures in the US (1). It is of paramount importance to delineate the molecular mechanisms of body mass control to develop new approaches to preventing and treating obesity.

Leptin, an adipocyte-secreted cytokine, is a key regulator of energy homeostasis, acting in large part by modulating neuroendocrine pathways that suppress food intake (FI) and increase energy expenditure (EE). Leptin affects several hypothalamic nuclei, including the arcuate nucleus (ARC), the ventromedial hypothalamus (VMH), and the dorsomedial hypothalamus (DMH) as well as extrahypothalamic sites (2). Deletion of the leptin receptor (Lepr) gene in all neurons leads to hyperphagia and severe obesity (3). In contrast, deletion of Leprin specific subsets of neurons, such as neuropeptide Y/agouti-related protein (NPY/AGRP) or proopiomelanocortin (POMC) ARC neurons, causes only mild obesity $(3,4)$. Thus, although leptin action on ARC neurons contributes to the regulation of energy stores and thereby adiposity, other neurons must also be involved in the full effect of leptin on adiposity.

Several lines of evidence indicate that the $\mathrm{VMH}$ is another important site for leptin action and the regulation of FI and EE (5).

Authorship note: Franck Chiappini and Karyn J. Catalano contributed equally to this work. Conflict of interest: The authors have declared that no conflict of interest exists. Submitted: May 5, 2014; Accepted: June 19, 2014.

Reference information: J Clin Invest. 2014;124(9):3781-3792. doi:10.1172/JCI68585.
Destruction of the VMH causes marked obesity $(6,7)$. Leptin infusion into the VMH suppresses FI and stimulates the sympathetic nervous system (SNS) (8-10). Lepr ablation in steroidogenic factor-1 (SF-1) neurons, the predominant type of neurons in the $\mathrm{VMH}$, results in mild obesity $(11,12)$, whereas Lepr deletion in POMC and SF-1 neurons together produces an additive increase in body weight that is still less than that in mice with whole-brain Lepr deficiency (12). Taken together, these data suggest that SF-1 neurons play an important role in fat mass regulation by leptin.

Protein-tyrosine phosphatase $1 \mathrm{~B}$ (PTP1B) is a ubiquitously expressed negative regulator of leptin receptor signaling and its subsequent effects on energy homeostasis $(13,14)$. РTP1B expression is increased in muscle, fat, and hypothalamus of obese rodents and humans (15-20), consistent with a role in leptin resistance. Whole-body Ptpn1 (the gene encoding PTP1B) deletion in mice results in decreased body weight, leanness, and resistance to high fat diet-induced (HFD-induced) obesity (21). Studies of compound mutant mice that lack Ptpn1 and leptin suggest that body mass regulation by PTP1B is mediated significantly, although not entirely, by its ability to negatively regulate central nervous system (CNS) LEPR signaling (22). Consistent with this notion, brain-specific (Nestin-Cre) Ptpn1/-- mice show increased EE and enhanced leptin-evoked suppression of FI (23). Furthermore, PTP1B dephosphorylates the LEPR-associated kinase JAK2, which mediates leptin-stimulated STAT3 phosphorylation. Accordingly, Ptpn1-deficient mice and cells show enhanced STAT3 phosphorylation in response to leptin stimulation $(21,24)$. Notably, deletion of Ptpn1 selectively in POMC neurons leads to a small increase in leptin sensitivity and a minor 


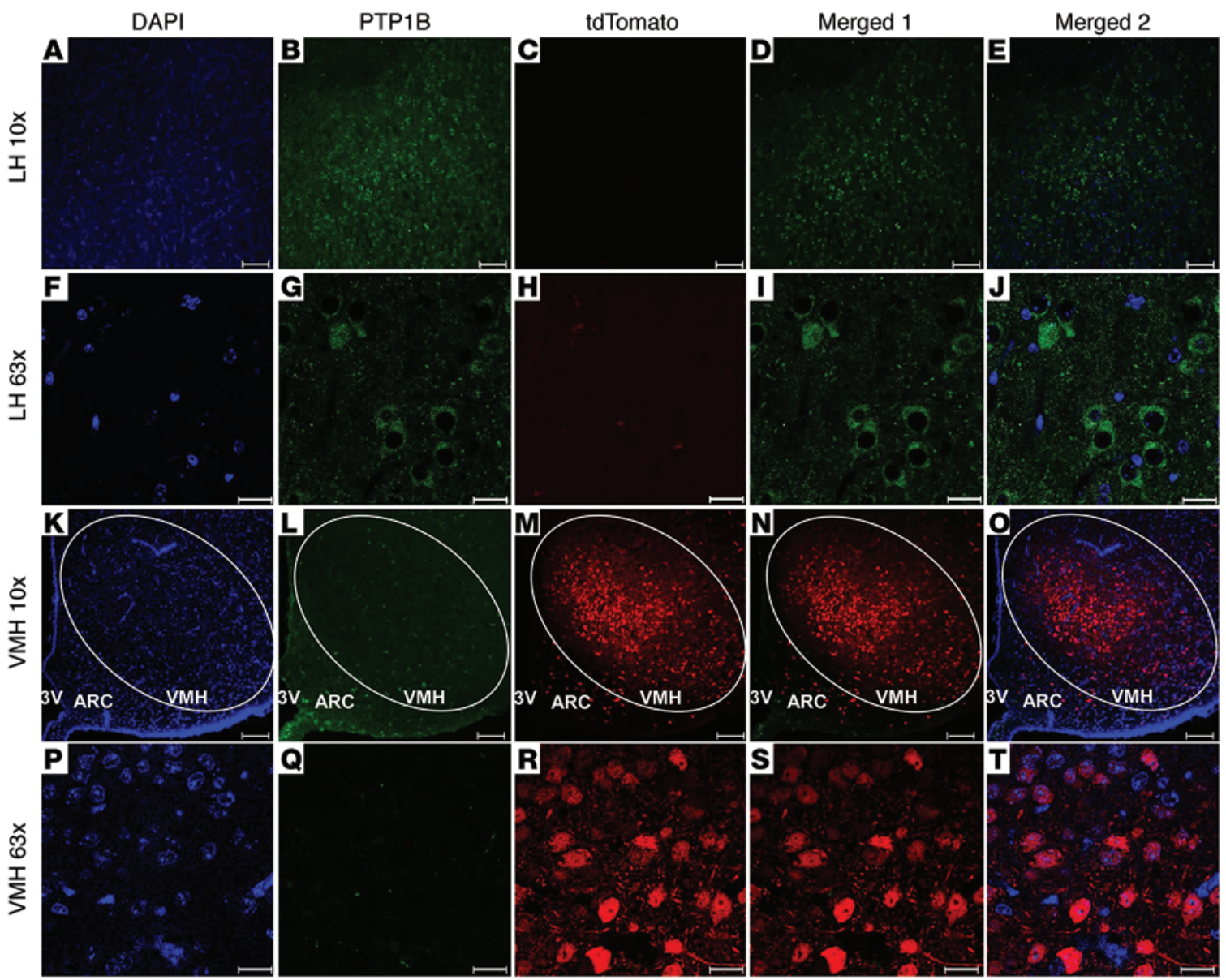

Figure 1. Selective ablation of PTP1B expression in SF-1 neurons of VMH of Sf1-Cre Ptpn1flox/flox Isl-tdTomato mice. Coronal sections of hypothalami from

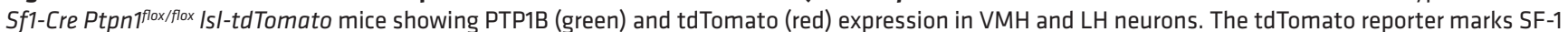
neurons in the VMH only. (A-J) Note that PTP1B is still present in tdTomato-negative LH neurons. (K-T) PTP1B protein is selectively absent in tdTomatopositive SF-1 neurons of the VMH. (P-T) Same sections at greater magnification. All immunofluorescence images are from the same brain. A, F, K, and $\mathbf{P}$ show cell nuclei stained with DAPI. Merged 1 shows overlay of anti-PTP1B (green) and tdTomato (red) fluorescence. Merged 2 shows overlay of DAPI, antiPTP1B (green), and tdTomato (red) staining. Scale bars: $100 \mu \mathrm{m}$ (A-E, K-0); $20 \mu \mathrm{m}$ (F-J, P-T). Original magnification: $\times 10$ (A-E, K-0); ×63 (F-J, P-T).

decrease in adiposity (25), suggesting that these neurons are responsible for some, but not all, of the effects of PTP1B on CNS leptin signaling and body fat mass.

PTP1B also is an important negative regulator of insulin receptor (INSR) signaling in peripheral tissues, where it dephosphorylates INSR and possibly downstream insulin receptor substrate (IRS) proteins (26-28). Mice with whole-body deletion of Ptpn1 have increased systemic insulin sensitivity $(27,29)$, even in the absence of altered adiposity (29). Furthermore, liver- or muscle-specific deletion of Ptpn1 increases INSR and IRS tyrosine phosphorylation, enhances whole-body insulin sensitivity, and improves glucose homeostasis without altering adiposity in mice on chow or HFDs $(30,31)$. Conversely, transgenic overexpression of PTP1B in muscle causes insulin resistance in mice (32), and restoring PTP1B expression in livers of whole-body $\mathrm{Ptpn1}^{-/-}$mice reduces their enhanced insulin sensitivity (33).

Mice with SF-1 neuron-specific INSR deficiency are protected against HFD-induced obesity and leptin resistance (34). Thus, insulin action in SF-1 neurons is also important for regulation of adiposity. Furthermore, deletion of the lipid phosphatase phosphate and tensin homolog (PTEN), a negative regulator of insulin action, in these neurons causes hyperphagia and increased adiposity. These data suggest that, in VMH neurons, INSR signaling regulates FI and energy homeostasis (34). Brain-specific (23) and POMC-specific (25) $\mathrm{Ptpn1}^{-/-}$mice have increased systemic insulin sensitivity. Similarly, rats with HFD-induced obesity (35) or with TNF- $\alpha$-induced leptin or insulin resistance (36) treated with Ptpn1 antisense oligonucleotide administered in their CNS exhibit decreased adiposity, improved whole-body insulin sensitivity, ameliorated leptin resistance, and augmented insulin-induced anorexigenic responses $(35,36)$. However, it is unclear which specific nucleus or nuclei are involved in these effects, as this treatment results in widespread downregulation of Ptpn1 in the hypothalamus. To determine whether PTP1B in the VMH regulates adiposity, and if so, which pathways are involved, we deleted Ptpn1 specifically in SF-1 neurons.

\section{Results}

$V M H$-specific PTP1B deletion. We first asked whether PTP1B could be detected in the VMH by means of immunofluorescence microscopy. Using anti-PTP1B antibodies, we detected specific immunostaining in the $\mathrm{VMH}$, as well as in the lateral hypothalamus (LH), of WT mice. Staining was absent in the same regions 
A
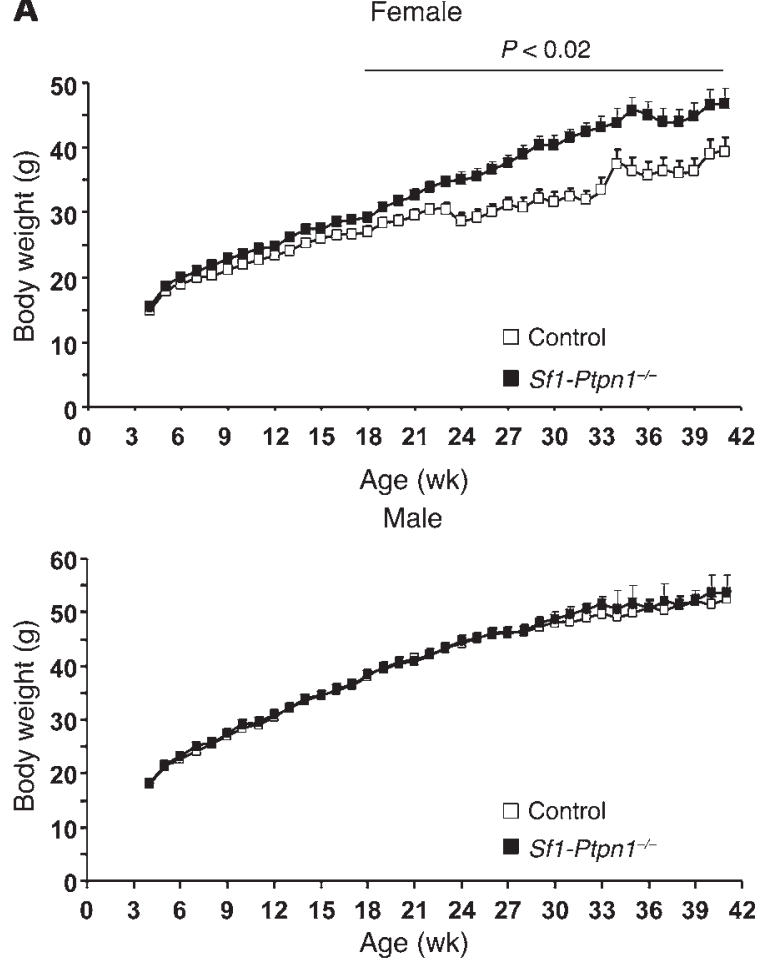

B
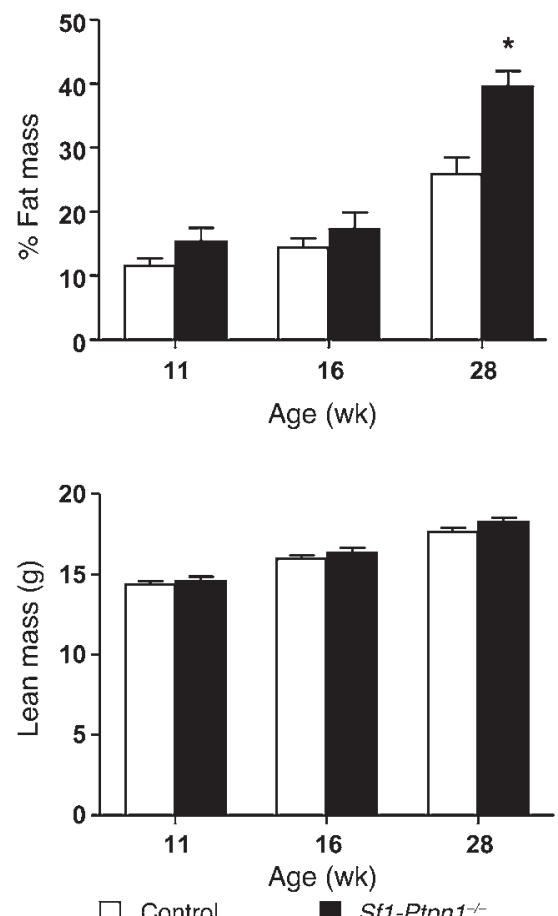

Figure 2. Effects of Ptpn1 deletion in SF-1-positive neurons on body mass regulation.

(A) Body weights of female and male Sf1-Ptpn 1-- and control (WT, Ptpn $1^{f l o x / f l o x}$, and Sf1-Cre mice) mice on HFD. (B) Body composition of female mice on HFD (control, $n=31$; Sf1-Ptpn1 1/-,$n=13$ ). (C) FI per mouse (left panel) and per gram body weight (right panel) in female mice on HFD at different ages (control, $n=10-11$; Sf1-Ptpn $\left.1^{-1-}, n=4\right)$. BW $\Delta$, body weight divergence. Data represent mean \pm SEM. ${ }^{*} P<0.05$, unpaired $t$ test compared with controls at the same age.

C

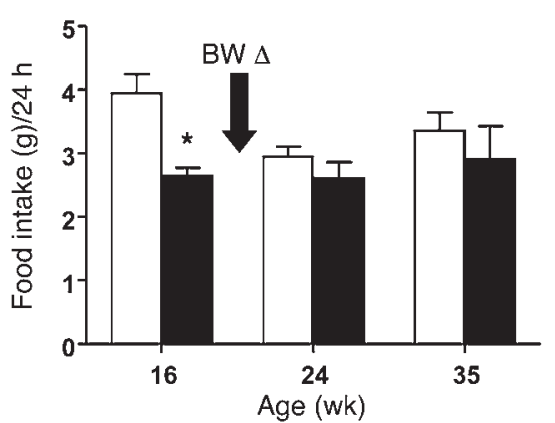

in global $\mathrm{Ptpn}^{-1-}$ mice, demonstrating that the antibodies were specific for PTP1B (Supplemental Figure 1; supplemental material available online with this article; doi:10.1172/JCI68585DS1). We also generated $S f 1$-Ptpn1 ${ }^{-/}$mice expressing tdTomato protein specifically in SF-1 neurons by crossing Ptpn $1^{f o x}{ }^{f f o x} l s l$-td-Tomato and Sf1-Cre mice (12). Sf1-Cre Ptpn $1^{10 x /+} l s l$-tdTomato mice were used to confirm the specificity of Sf1-Cre expression in the VMH. Supplemental Figure 2 shows that PTP1B is present in VMH neurons of Sfl-Cre Ptpn $1^{f o x /+} l$ sl-tdTomato mice and colocalizes with tdTomato, which is visualized when SfI-Cre removes the stop codon.

In contrast, and as expected, PTP1B was selectively absent in tdTomato-positive neurons in the VMH of Sf1-Cre Ptpn $1^{f o x / f l o x}$ lsl-tdTomato, while in LH neurons from the same mice, PTP1B expression was normal, and tdTomato was not expressed (Figure 1). These data demonstrate that, as shown for other floxed alleles (12, 34, 37-39), Sf1-Cre drives selective deletion of Ptpn1 in SF-1 neurons of the VMH.

Female Sf1-Ptpn1-/ mice on HFD develop greater obesity than controls. Next, we generated cohorts of Sf1-Ptpn1 ${ }^{-1-}$ mice and littermate controls $\left(P t p n 1^{+/+}\right.$, Ptpn $1^{f l o x f f o x}$, and Sf1-Cre Ptpn $\left.1^{+/+}\right)$weaned onto chow or HFDs. On chow, Sf1-Ptpn1-- mice and controls had similar body weight (Supplemental Figure 3) and FI (data not shown). In contrast, on HFD, female Sf1-Ptpn1 1/- mice gained more weight than controls, beginning at 15 to 16 weeks of age (Figure $2 \mathrm{~A})$. The increase in body weight was due entirely to increased adiposity (Figure 2B). Increased body weight and adiposity were not seen in male Sf1-Ptpn1-/- mice (Figure 2, A and B).

The $S f-1$ gene is expressed in the adrenal, pituitary, and gonads in addition to the $\mathrm{VMH}$, and altered signaling in these glands could contribute to changes in adiposity. Altered gonadal function is unlikely to account for greater adiposity in Sf1-Ptpn $1^{-1-}$ mice, as serum estradiol and testosterone levels were similar to those in controls of both sexes (Supplemental Figure 4, A and B). We also assessed reproductive function by mating male or female Sf1-Ptpn1- mice with Ptpn $1^{f o x}{ }^{f / f o x}$ mice. No differences in fecundity were observed when either parent was a Sf1-Ptpn1 ${ }^{-/}$mouse (Supplemental Figure 4, C and D). Likewise, basal and stress-induced corticosterone levels were indistinguishable in control and SfI$\mathrm{Ptpn1}^{-/}$mice (Supplemental Figure 4, E and F, respectively), indicating preserved adrenal function. Thyroid function, assessed by 

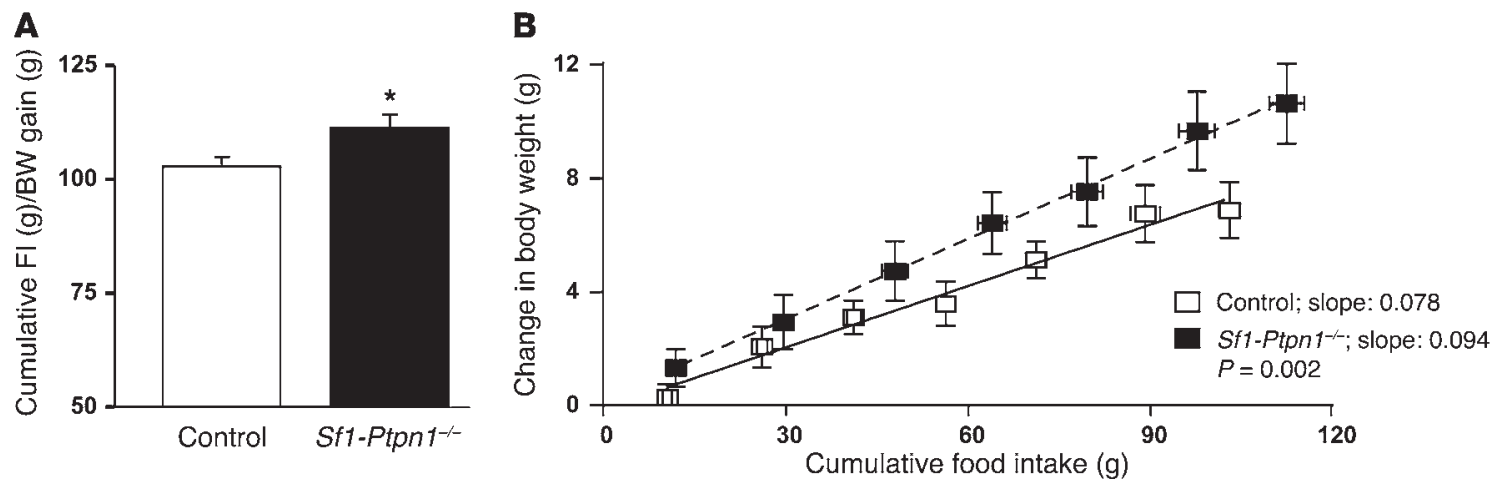

Figure 3. Female Sf1-Ptpn1-/- mice have increased feed efficiency. (A) Cumulative Fl (g/mouse) over 7 weeks divided by the cumulative weight ( $\mathrm{g}$ ) gained over the same time period in 15- to 16 -week-old female mice on HFD; $n=7$ per group. Data shown represent mean $\pm \mathrm{SEM}$. ${ }^{*} P=0.016$ by unpaired $t$ test.

(B) Change in body weight versus cumulative $\mathrm{Fl}$ in the same mice. Slopes are compared by ANCOVA. Data represent mean \pm SEM.

total serum T4 levels, also was normal in Sf1-Ptpn1 $1^{-1-}$ female mice on HFD (Supplemental Figure 4G). These data indicate that the observed adiposity phenotype in these mice is highly likely to be due to the absence of PTP1B in SF-1 neurons.

Obesity is often caused by increased FI. We measured 24-hour FI in female control and Sf1-Ptpn1-- mice prior to (16 weeks) and after (24 weeks) body weight divergence and at a more advanced age (35 weeks). Surprisingly, before the body weight divergence, Sf1-Ptpn1-- mice ate less than controls (Figure 2C). Once Sf1Ptpn1-- mice became obese, their absolute FI (g/mouse per day) was not different compared with that of control mice. However,

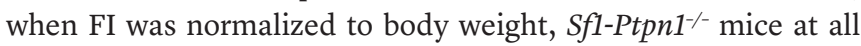
ages ate less than controls. Thus, the increased obesity of SfIPtpn 1-/ mice on HFD cannot be explained by increased FI.

Sf1-Ptpn1- mice have decreased EE. The increased body mass/ adiposity in the presence of decreased FI in Sf1-Ptpn1 $1^{-/}$mice suggested that their energy balance was altered. We measured feed efficiency (increase in body weight/FI) for 7 weeks during the period in which body weight diverged. Indeed, feed efficiency was higher in female Sf1-Ptpn1-/ mice (Figure 3), which likely explains, at least in part, their increased weight gain on HFD. To confirm these results, we also measured feed efficiency during fasting/refeeding in a separate set of control and Sf1-Ptpn1-/ mice before and after body weight divergence. For these experiments, mice were fasted for 24 hours, and then FI and body weight were measured over a 24-hour refeeding period. Again, feed efficiency was increased in Sf1-Ptpn1-mice compared with controls prior to (Supplemental Figure 5A) and after (Supplemental Figure 5B) body weight divergence.

These findings suggested that the enhanced obesity in $S f_{1-}$ Ptpn1 $^{--}$mice was due to decreased EE. To test this possibility directly, we used indirect calorimetry to measure $\mathrm{VO}_{2}$ and $\mathrm{EE}$.

Figure 4. Decreased EE in female Sf1-Ptpn1/- mice. (A) Mean volume of $\mathrm{O}_{2}$ consumed $\left(\mathrm{VO}_{2}\right.$; left) and $\mathrm{EE}$ (right) during the dark phase, normalized to lean body mass (LBM), as determined by ANCOVA per a previously published analytical protocol (54). (B) Mean $\mathrm{VO}_{2}$ (left) and $\mathrm{EE}$ (right) during the resting period, normalized to lean body mass. Resting periods are defined as periods with activity counts of less than $5 \%$ of maximum. Studies were performed in 16- to 17-week-old female mice on HFD. Controls, $n=5$; Sf1Ptpn $1^{-1}, n=8$. Bar graphs represent mean \pm SEM. ${ }^{*} P<0.05$ compared with control mice by unpaired $t$ test.
Consistent with the feed efficiency data, both were reduced in SfIPtpn1-- mice compared with controls (Figure 4). In addition, spontaneous locomotor activity was reduced by approximately $75 \%$ in Sf1-Ptpn1-- mice (Figure 5, A and B). A similar decrease $(\sim 80 \%)$ was observed in Sf1-Ptpn1 ${ }^{-\digamma}$ mice that were weight matched with controls (Figure 5C). Thus, the lower EE in Sf1-Ptpn1-/- mice could be due, at least in part, to decreased spontaneous locomotor activity. Sf1-Ptpn1-- mice had a lower core body temperature (CBT) at room temperature (RT) and a larger nocturnal decrease in CBT compared with control mice (Figure 6A). Upon chronic cold exposure, CBT of Sf1-Ptpn1 $1^{--}$mice was comparable to that of controls. However, maintenance of CBT following acute (4 hours) cold exposure $\left(4^{\circ} \mathrm{C}\right)$ was markedly impaired in Sf1-Ptpn1 $1^{-/}$mice before it ultimately stabilized (Figure 6B). Sf1-Ptp1 $b^{-/}$mice also ate less (Figure 6C) and lost more weight (Figure 6D) during cold exposure.

Taken together, these data suggest an impaired sympathetic response to cold exposure in Sf1-Ptp1 $b^{-/-}$mice. Therefore, we analyzed genes critical for thermoregulation in brown adipose tissue (BAT). When mice were maintained at RT, uncoupling protein 1
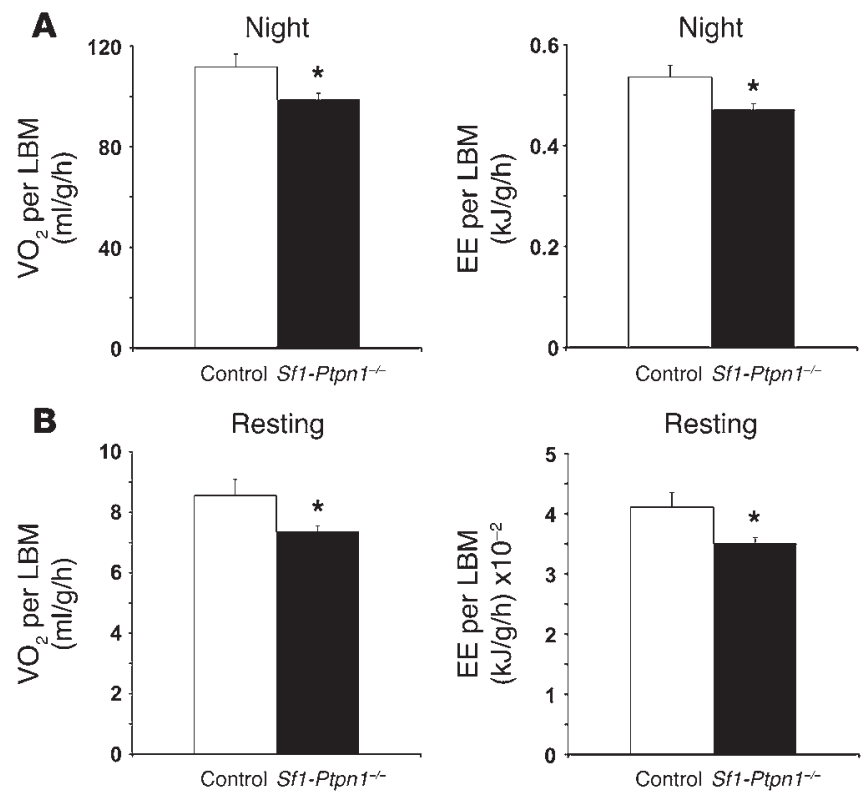
A

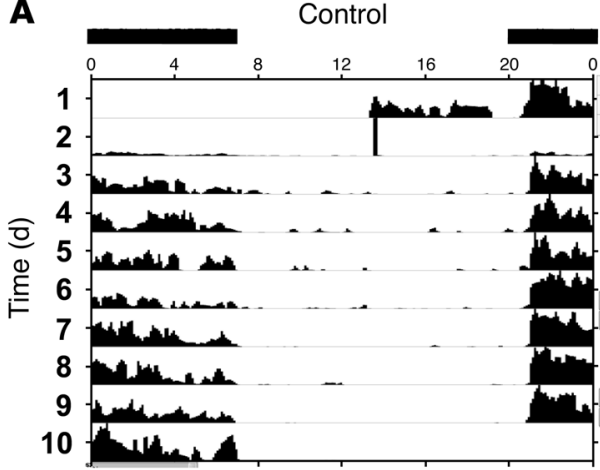

B

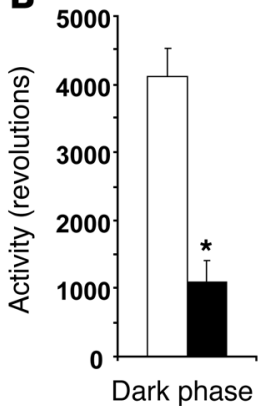

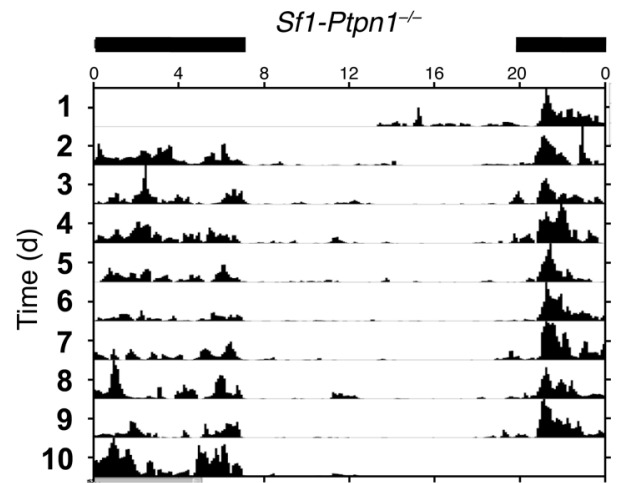

C

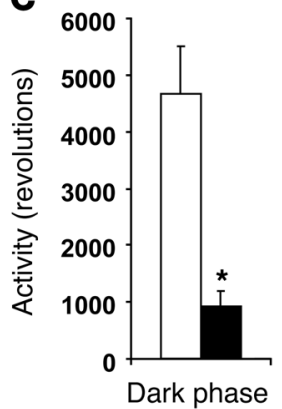

Figure 5. Decreased spontaneous activity in female Sf1-Ptpn 1-/- mice. (A) Actogram showing voluntary activity in control (left) and Sf1-Ptpn1 1/- (right) mice during 10 days of housing with a running wheel. Horizontal black bars above actograms indicate dark periods (control, $n=8$; Sf1Ptpn1 1/-,$n=5$ ). (B) Average daily activity during dark phase (10 hour) averaged over the 10-day period (white bar, control $n=8$; black bar, Sf1-Ptpn 1/-, $n=5$ ). (C) Average activity of a subset of weight-matched Sf1-Ptpn1 1-- (black bar, $n=4$ ) and control (white bar, $n=3$ ) mice. All mice were 33to 35 -week-old females on HFD. ${ }^{*} P<0.05$ by unpaired $t$ test.
(Ucp1) mRNA levels were lower in BAT of Sf1-Ptpn1-/- mice compared with controls. Following acute cold exposure, however, Ucp1 mRNA (Figure 6E) and protein (Figure 6F) levels in BAT increased to levels similar to those in Sf1-Ptpn1 ${ }^{-/}$and control mice. Ucp2, Ucp3, and Pgc1 $\alpha$ expression levels were indistinguishable in WT and Sf1-Ptpn1 $1^{-1-}$ mice maintained at RT or subjected to cold (Supplemental Figure 6, A-C). Serum norepinephrine levels, an indicator of SNS activity, were lower in Sf1-Ptpn1 ${ }^{-/-}$mice at RT compared with controls, but these increased to equivalent levels in both genotypes upon cold exposure (Figure 6G). Thus, our data indicate that sympathetic tone is impaired in Sf1-Ptpn1-/- mice at baseline but not in response to cold.

Sf1-Ptpn1 $1^{-/}$mice on HFD have increased leptin sensitivity. Typically, obesity is accompanied by leptin resistance and hyperleptinemia (40). Indeed, serum leptin levels (Figure 7A) and leptin mRNA in perigonadal white adipose tissue (WAT) (Figure 7B) were elevated in obese female Sf1-Ptpn1/-- mice compared with controls. However, leptin sensitivity tests using a submaximal leptin dose $(1.5 \mathrm{mg} / \mathrm{kg}$ i.p. twice daily for 3 days) revealed enhanced suppression of FI in Sf1-Ptpn1 $1^{-/}$mice (Figure 7C). Similar results were obtained when mice were given a lower dose of leptin $(0.75 \mathrm{mg} / \mathrm{kg}$ i.p. twice daily) over 3 days (data not shown). In addition, at a dose of leptin that failed to produce a change in $\mathrm{O}_{2}$ consumption in control mice, $\mathrm{O}_{2}$ consumption was increased in Sf1-Ptpn1 ${ }^{-/-}$mice (Figure 7D).

We sought to determine the leptin signaling steps that were affected by absence of PTP1B in SF-1 neurons. PTP1B is a key negative regulator of leptin-induced STAT3 phosphorylation (24). Accordingly, there was a 3.5-fold increase in the number of PSTAT3positive neurons in the VMH of Sf1-Ptpn1 $1^{-/}$mice (Figure 7E). This increase is likely to result from the combined effects of PTP1B deficiency in VMH neurons and increased circulating leptin levels in these mice. Despite the increased leptin levels, we did not observe an increase in pSTAT3-positive neurons in the ARC of Sf1-Ptpn $1^{-/-}$mice. Therefore, the increased STAT3 activation in the VMH of Sf1-Ptpn1 1/- mice likely explains their reduced FI. Yet despite their obesity and hyperleptinemia, which is often associated with leptin resistance in the setting of obesity, Sf1-Ptpn1-/- mice are hypersensitive to the effects of leptin on FI and body fat accumulation (Figure 7, C and E). This finding makes their increased obesity even more unexpected.

Sf1-Ptpn1-1- female mice exhibit insulin hypersensitivity in VMH. In addition to leptin-responsive neurons, the VMH has neurons that express INSR, and recent work indicates that INSR signaling in these neurons suppresses EE (34). Because PTP1B negatively regulates the INSR in multiple peripheral tissues, we hypothesized that the decreased EE and increased body mass in Sf1-Ptpn1-/- mice might reflect an augmented insulin response in these $\mathrm{VMH}$ neurons.

To test this possibility, we injected insulin or vehicle (artificial cerebrospinal fluid [aCSF]) directly into the $\mathrm{VMH}$ of control and Sf1-Ptpn1 $1^{--}$mice. As expected, control aCSF injections did not induce phosphorylation of AKT or FOXO1 in VMH neurons (data not shown). In contrast, following injection of a low dose of insulin, AKT and FOXO1 phosphorylation were increased in the VMH of Sf1-Ptpn1/-, but not control, mice (Figure 8, A-C). As expected, the contralateral (uninjected) VMH showed no increase in insulin signaling (Figure 8, A and B). Sf1-Insr-- mice have increased EE (34); hence, enhanced insulin signaling in the VMH of Sf1-Ptpn1 ${ }^{-/-}$ mice could explain their reduced EE and obesity.

In contrast to the increased insulin sensitivity in the $\mathrm{VMH}$, systemic insulin responsiveness was modestly decreased in Sf1-Ptpn1-/mice on HFD. Glucose and insulin levels were elevated 5 hours after food removal (Supplemental Table 1). Furthermore, although glucose tolerance was similar between SF-1-Ptpn1--- and control mice, insulin secretion in response to the same glucose load was higher in Sf1-Ptpn1 $1^{-/-}$mice when compared with controls (Supplemental Figure 7). These results indicate mild total body insulin resistance in Sf1-Ptpn1 $1^{-/-}$mice, consistent with their increased adiposity. 
A

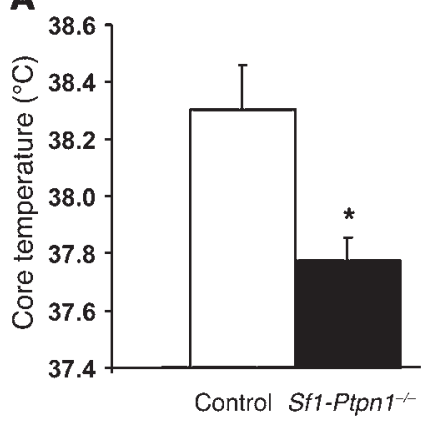

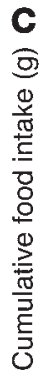

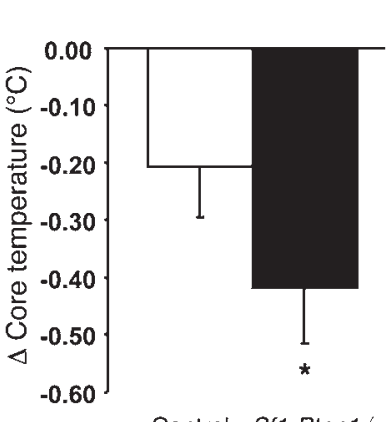

Control Sf1-Ptpn 1t
B

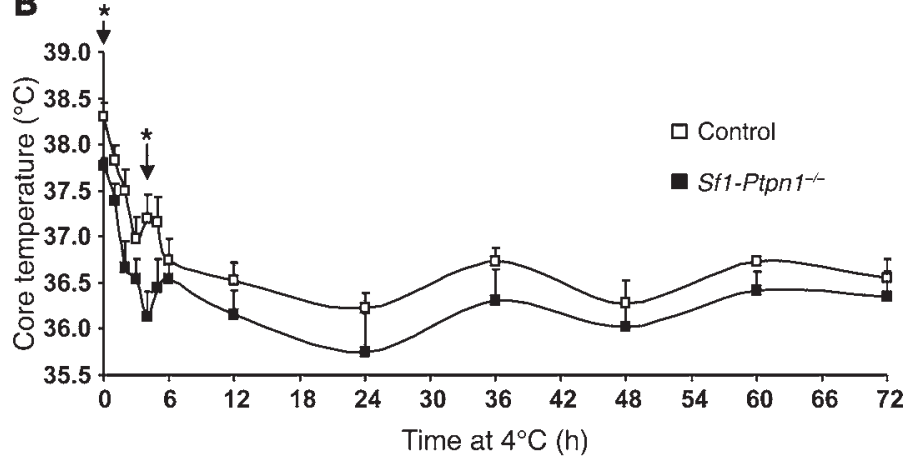

D

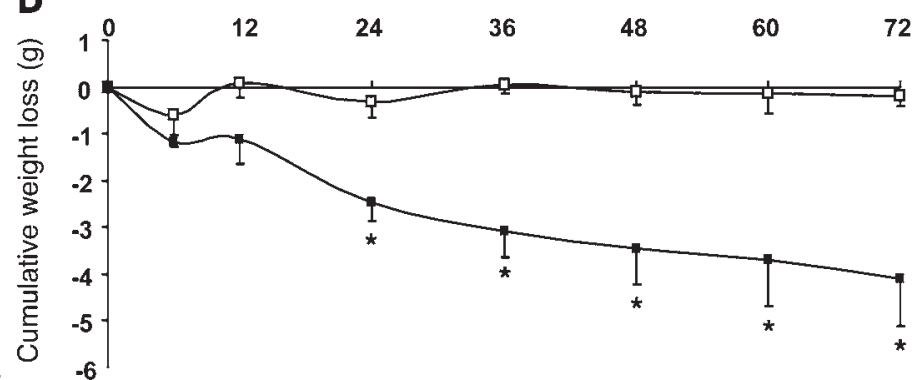

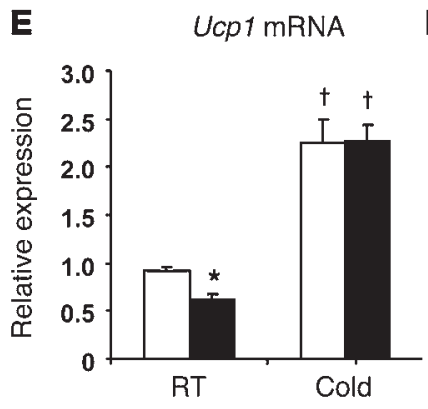

$\mathbf{F}$

F UCP1 protein

G
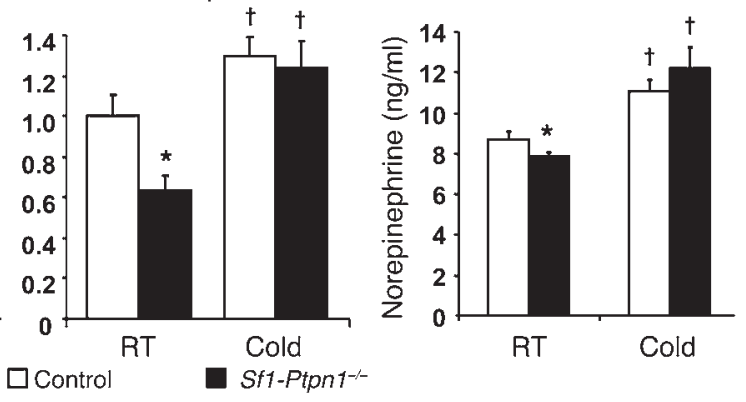

Figure 6. Altered sympathetic tone in Sf1-Ptpn1-- mice. (A) CBT at 8 am (left) and change in CBT between morning and evening (right) in mice maintained at RT. (B) CBT, (C) cumulative FI, and (D) cumulative weight loss during cold exposure ( $\left.4^{\circ} \mathrm{C}\right)$. (E) Ucp1 mRNA (normalized to $18 \mathrm{~S}$ RNA) and (F) protein levels (normalized to GAPDH, quantified by Western blot) in BAT at RT and after 3 days of cold exposure $\left(4^{\circ} \mathrm{C}\right)$. (C) Serum norepinephrine levels in ad libitum-fed mice. All experiments were performed on female mice on HFD at 36 to 38 weeks of age. Controls, $n=8 ;$ Sf1-Ptpn $1^{-1-}, n=5$. Data represent mean \pm SEM. ${ }^{*} P<0.05$ compared with control at RT. ${ }^{\dagger} P<0.05$ compared with the same genotype at RT. All comparisons are by unpaired $t$ test.

Effects of estrogen on energy homeostasis in Sf1-Ptpn1-1- female mice. Estrogen signaling in the VMH of female mice contributes to control of energy balance through changes in EE and SNS activity (38). Nearly $50 \%$ of estrogen receptor $\alpha$-positive (ER $\alpha$ positive) neurons in the VMH coexpress SF-1, whereas approximately $12 \%$ of SF- 1 neurons express $\mathrm{ER} \alpha(38,41)$. To test whether altered estrogen signaling might play a role in the apparent sexual dimorphism in Sf1-Ptpn1-- mice on HFD, we implanted subcutaneous estradiol-17 $\beta$ pellets into male mice on HFD. Estrogen treatment caused a significant reduction in body weight (Supplemental Figure 8A) and feed efficiency (Supplemental Figure $8 \mathrm{~B}$ ) without altering FI (data not shown) in controls and Sf1-Ptpn1-- male mice. In contrast, depleting estrogen by ovariectomy (Figure 9A) caused marked weight gain in controls, but not in Sf1-Ptpn 1/- female mice (Figure 9B). These effects could not be explained by differences in ER $\alpha$ or ER $\beta$ expression in ARC, paraventricular nuclei (PVN), or DMH/VMH (Supplemental Figure 9). Furthermore, the failure of ovariectomy to enhance the effects of PTP1B deficiency suggests that PTP1B and estrogen act in the same pathway in SF-1 neurons to regulate adiposity in older female mice.

\section{Discussion}

PTP1B is a critical negative regulator of leptin action in the brain and of insulin signaling in peripheral tissues $(13,14)$. Ptpn1 inhibition and/or deletion in the brain enhances leptin signaling and thus improves energy balance and glucose homeostasis $(21,23$, $25,27,29)$. Ptpn1 deletion in muscle and liver enhances insulin action in these tissues and also increases systemic insulin sensitivity systemically $(30,31)$, whereas PTP1B overexpression has the opposite effects $(15-20,32,33)$. INSRs and LEPRs are coexpressed in several hypothalamic nuclei, including the VMH (42). Several studies have shown that insulin administration in the CNS can modulate FI (43-47) and that VMH-specific Insr deletion causes weight loss in mice (34). Hence, elucidating the role of PTP1B in VMH regulation of energy balance is of interest. 
A

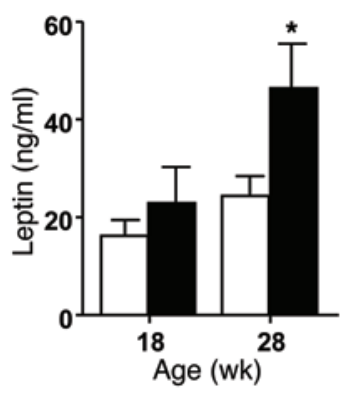

B

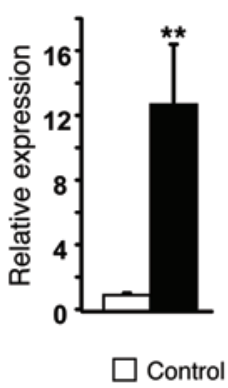

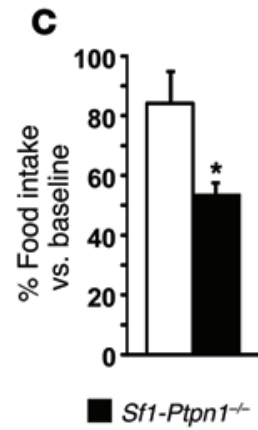

D

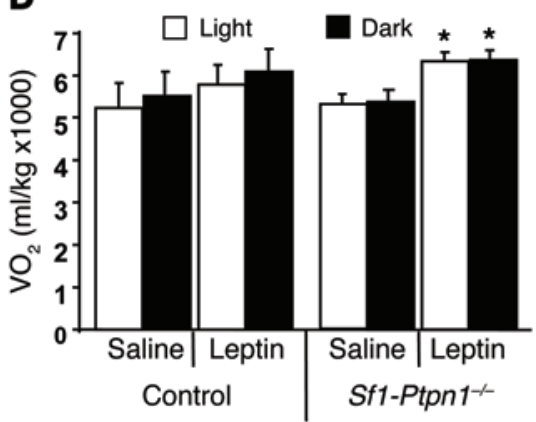

E

Control

Sf1-Ptpn 1-
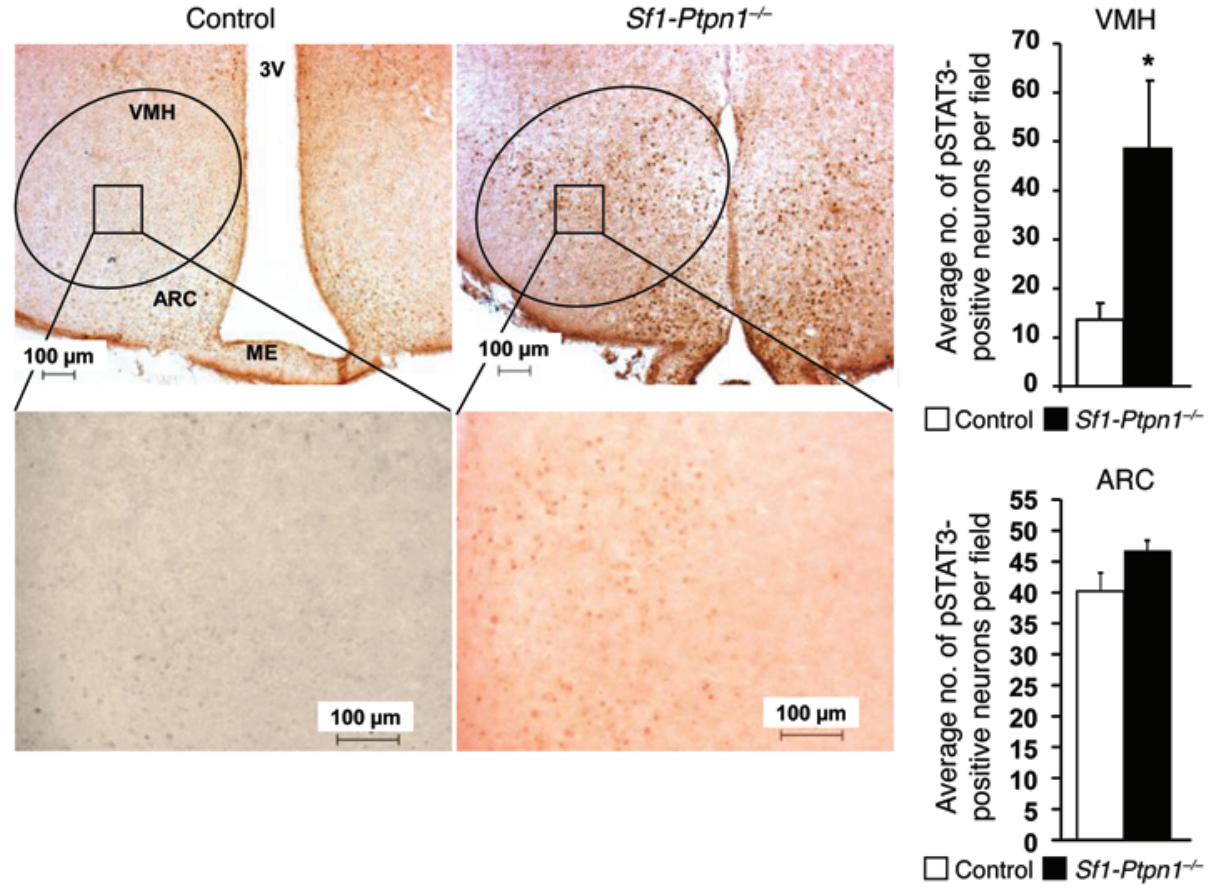

Figure 7. Increased leptin levels, leptin sensitivity, and leptin signaling in female Sf1-Ptpn1 ${ }^{-1-}$ mice on HFD. (A) Serum leptin levels at 18 and 28 weeks of age. (B) Leptin mRNA levels (normalized to 18S) in WAT from 28-week-old mice. Controls, $n=21 ; 5 f 1-P t p n 1^{-1-}, n=9$. (C) FI (as percentage of baseline) following leptin injections (1.5 mg/kg i.p.) twice a day for 3 days. Baseline FI was measured over the 3 days prior to the experiment, during which mice received saline only. (D) Oxygen consumption ( $\left.\mathrm{VO}_{2}\right)$ at baseline and after leptin injection ( $5 \mathrm{mg} / \mathrm{kg}$ i.p.; 3 doses at 12-hour intervals) in fed, 18-week-old mice. $n=8$ per genotype. Data represent mean \pm SEM. ${ }^{*} P<0.05$; ${ }^{* *} P<0.01$, by unpaired $t$ test (A-C) or paired $t$ test (D). (E) pSTAT3 immunostaining in control $(n=9)$ and Sf1-Ptpn1-- $(n=8)$ mice in the VMH (top) and ARC (bottom). pSTAT3-positive neurons were quantified in 3 to 6 brain slices/mouse. Original magnification, $\times 10$ (upper panels); $\times 40$ (lower panels). Scale bars: $100 \mu \mathrm{m} .{ }^{*} P<0.05$, by unpaired $t$ test. $3 \mathrm{~V}$, third ventricle; ME, median eminence. Data represent means \pm SEM.

In the current study, we found that female mice lacking Ptpn1 in SF-1 neurons are, unexpectedly, more obese on a HFD compared with control mice, despite having enhanced leptin sensitivity. SF-1 is expressed in the adrenals, gonads, and pituitary as well as in the hypothalamus. However, the adiposity phenotype of $S f 1-P t p n 1^{-/}$mice clearly results from the absence of Ptpn1 in SF-1 neurons, as evidenced by unaltered glucocorticoid levels basally and in response to stress, normal thyroxine levels, and normal reproductive function in these mice. Although whole-brain Ptpn1 deficiency causes leanness (23) and Sf1-Ptpn1-- mice have leptin hypersensitivity with reduced FI (Figure 6), Sf1-Ptpn1 $1^{-1-}$ mice on HFD are more obese as a consequence of decreased EE. Leptin and insulin signaling in the VMH are increased in Sf1-Ptpn1 $1^{--}$mice, and the effects of estrogen on body weight are also impaired. Overall, our results demonstrate a role for PTP1B in modulating insulin signaling in the CNS. Moreover, our data suggest that the effects of Ptpn1 deficiency on insulin action in the $\mathrm{VMH}$ override those of increased leptin signaling. Likewise, the effects of PTP1B deficiency on EE dominate over the decreased FI, ultimately leading to enhanced obesity.

The decreased FI in SfI-Ptpn1-- mice can be explained in at least 2 ways. Sf1-Ptpn1-/ mice show evidence of enhanced leptin signaling in the VMH: at ambient leptin levels, the number of pSTAT3-positive neurons is increased relative to controls. The fact that leptin action on VMH neurons regulates FI is well documented $(48,49)$; hence, increased leptin sensitivity in these neurons probably contributes to the markedly reduced FI in Sf1-

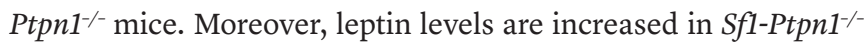
mice, especially as their body weight begins to diverge from that of controls. Leptin mRNA levels in WAT are also augmented, 
A

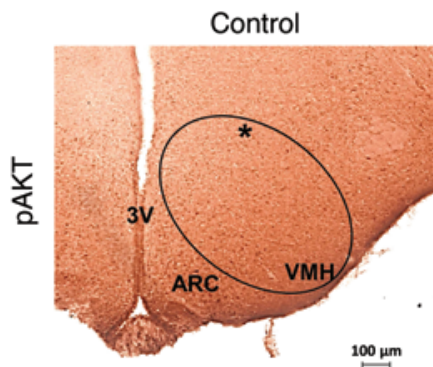

B
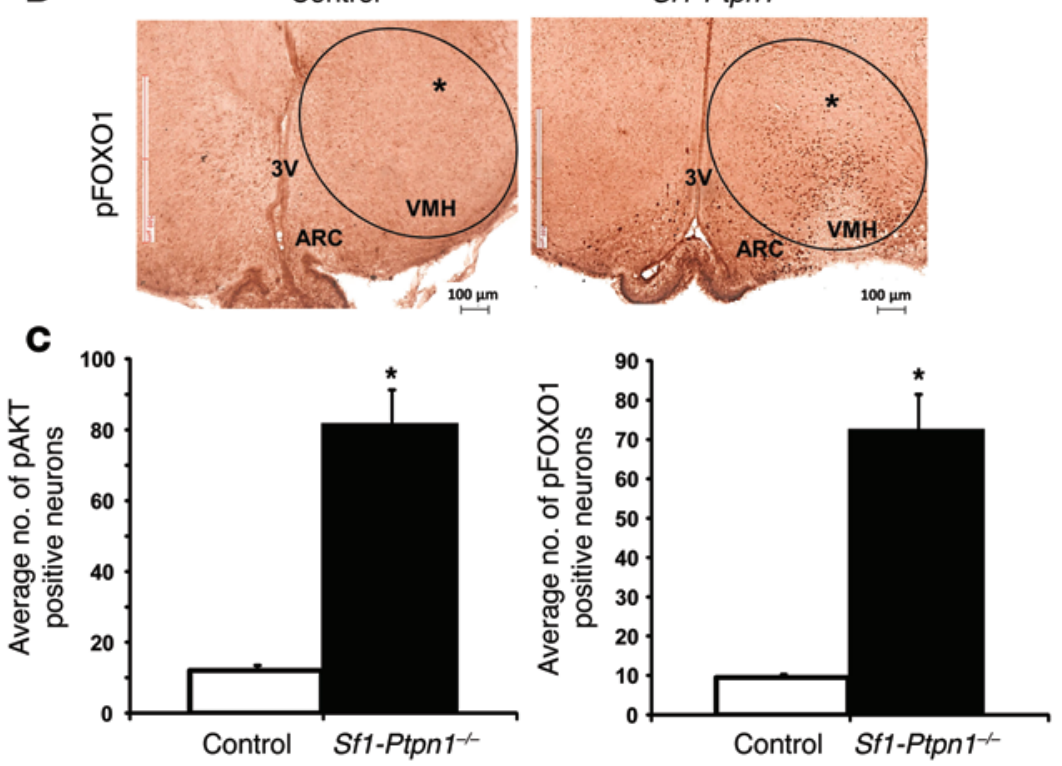

Figure 8. Increased insulin response in VMH of Sf1-Ptpn1/- mice on HFD. (A) pAKT and (B) pFOX01 immunostaining following injection of insulin ( $4 \mu \mathrm{U} / \mu \mathrm{l}$ in aCSF) into the $\mathrm{VMH}$. Asterisk indicates location of cannula. (C) The numbers of pAKT- and pFOXO1-positive neurons were quantified in 3 to 6 slices per brain from 3 different brains per genotype, injected with either aCSF or insulin unilaterally in the VMH. Neither aCSF-injected nor uninjected VMH showed significant staining (data not shown). Bars represent means $\pm \mathrm{SEM} ;{ }^{*} P<0.05$, unpaired $t$ test. Circles indicate VMH. Scale bars: $100 \mu \mathrm{m}$. Original magnification: $\times 10$ (A and $\mathbf{B}$ ).

indicating that elevated circulating leptin reflects increased synthesis/secretion rather than impaired clearance. Previously, we found that neuronal Ptpn1-/- mice (generated using Nestin-Cre) have increased circulating leptin levels, even though their body weight and fat mass are decreased (23). Our finding that leptin levels are increased in Sf1-Ptpn1-/- mice suggests that Ptpn1 deficiency in VMH neurons could contribute to the hyperleptinemia seen in neuronal Ptpn1 ${ }^{-/}$mice. However, we cannot exclude the possibility that in Sf1-Ptpn1-/ mice, the increased leptin levels simply reflect their increased adiposity. Indeed, serum leptin levels correlated strongly with increased fat mass in these animals (data not shown). Overall, the increased leptin levels in Sf1-Ptpn1in the setting of enhanced leptin responsiveness of SF-1 neurons in the VMH likely accounts for the observed reduction in FI.

Despite their augmented leptin sensitivity/signaling and reduced FI, Sf1-Ptpn1 1- mice develop increased adiposity on HFD compared with controls. Our data show clearly that decreased $\mathrm{EE}$ underlies this difference: feed efficiency is increased and $\mathrm{O}_{2}$ consumption is decreased. Reduced EE, in turn, is due, at least in part, to decreased locomotor activity. However, Sf1-Ptpn1 ${ }^{-/}$mice also have decreased CBT, serum norepinephrine levels, and UCP1 expression in BAT, consistent with decreased sympathetic tone. Increased insulin sensitivity in the VMH might explain these effects, as microinjection of insulin into the $\mathrm{VMH}$ suppresses the firing rate of sympathetic neurons (50).

The energy utilization defect in Sf1-Ptpn1-/mice differs dramatically from the effects of Ptpn1 deficiency in whole brain, in POMC neurons, and in all leptin receptor-expressing neurons $(23,25,51)$. Whereas one might expect leanness to result from the increased leptin sensitivity in Sf1-Ptpn1/-- mice, instead, their phenotype is unexpectedly similar to the effects of SF-1 neuron-specific Lepr deletion $(11,12)$. In contrast, the obesity and decreased EE phenotype in Sf1-Ptpn1 $1^{-/}$mice is consistent with several models in which genes encoding components of the insulin-signaling pathway have been deleted in SF-1 neurons. For example, deletion of Insr or Foxo1 in SF-1 neurons results in leanness (34, 52). Conversely, deletion of Pten, another negative regulator of insulin signaling, results in obesity (34), similar to the effects of SF-1 deletion of Ptpn1. However, the leptin and insulin-signaling pathways share several components, which can result in unanticipated complexities and/or crosstalk. For example, deletion of the gene encoding the $p 110 \alpha$ catalytic subunit of PI3K also results in obesity (53). In addition, SF-1-specific Insr deletion protects against diet-induced leptin resistance (34). Notably, VMH-specific deletion of Socs-3, another wellknown inhibitor of leptin and insulin signaling, did not result in a change in body weight (39). Thus, it is likely that Ptpn1 deletion in SF-1 neurons increases both leptin and insulin signaling, but these effects compete with each other (e.g., decreased FI vs. decreased EE, respectively). Apparently, over time, suppressed EE prevails over decreased FI, resulting in obesity. Notably, altered body fat occurs earlier in Sf1-Insr ${ }^{--}$or Sf1-Foxo $1^{-/-}$ mice than in our model. However, INSR and FOXO1 mediate insulin signaling, whereas PTP1B is a modulator of this pathway, likely yielding less pronounced changes in $\mathrm{EE}$ and a more delayed (15-16 weeks) onset of enhanced adiposity $(34,54)$.

A subset of SF-1-positive neurons in the VMH express ERs (42). These neurons could play a role in the control of FI and EE because they communicate directly with LEPR-and INSR-positive VMH neurons $(5,48,55,56)$ and estrogen deficiency causes weight gain. We observed an apparent sexual dimorphism in weight gain with VMH-specific deletion of Ptpn1. The effects of PTP1B deficiency on adiposity in female mice cannot be explained by altered ER $\alpha$ or ER $\beta$ levels (Supplemental Figure 9). In addition, ovariectomy did not enhance the weight gain seen in Sf1-Ptpn1-/ mice on HFD (Figure 9), indicating that lack of estrogen and PTP1B are not additive and, therefore, estrogen and PTP1B likely act in the same pathway to control body weight.

In conclusion, in Sfl-expressing VMH neurons, as in ARC POMC neurons, PTP1B is a critical negative regulator of the INSR and LEPR pathways. In ARC POMC neurons, INSR and LEPR 
A

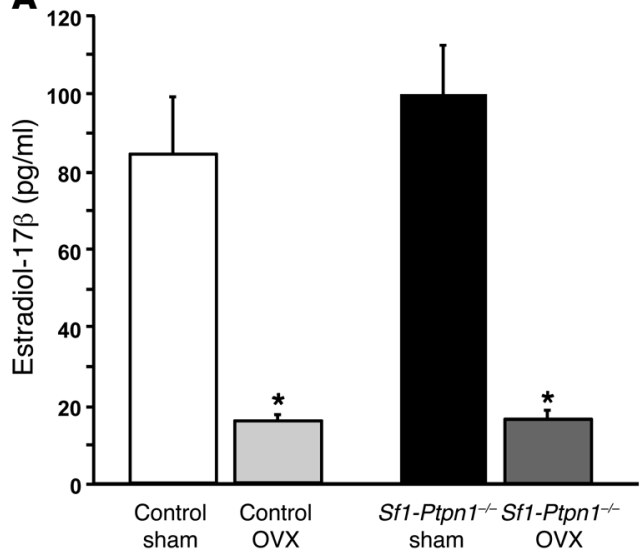

B

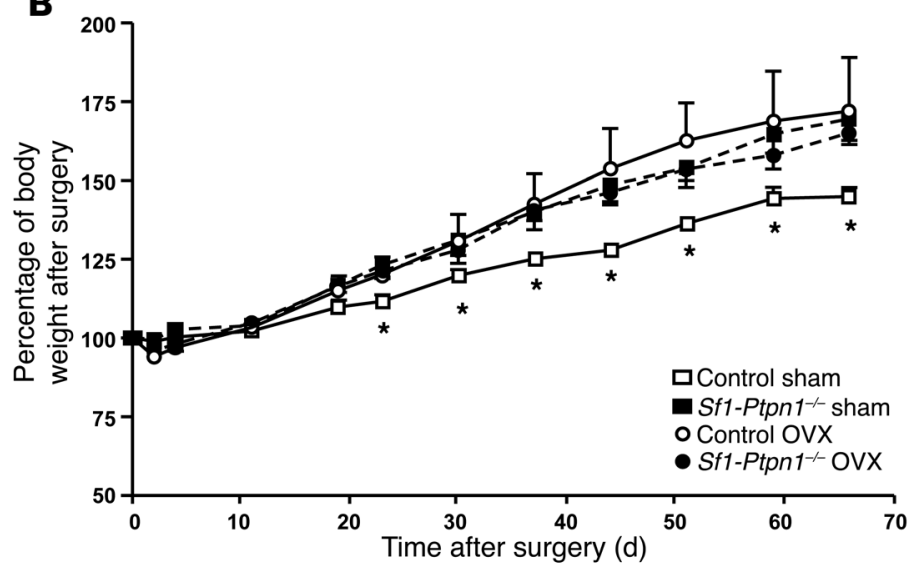

Figure 9. Ovariectomized Sf1-Ptpn1 ${ }^{-/-}$mice do not gain additional weight on HFD. (A) Estradiol-17 $\beta$ serum levels in female sham or ovariectomized (OVX) control and Sf1-Ptpn1/- mice on HFD, measured 66 days postoperatively. Data represent mean $\pm \mathrm{SEM}$. ${ }^{*} P<0.05$, unpaired $t$ test compared with respective controls. (B) Percentage of body weight (weight gain) after surgery in control sham (open squares), 5f1-Ptpn1 ${ }^{-1-}$ sham (black squares), control ovariectomized (open circles), and Sf1-Ptpn1 1/- ovariectomized (black circles) mice on HFD, respectively. Control $(n=5)$; Sf1-Ptpn1 $1^{-1-}(n=7)$. Data are shown as mean \pm SEM. Groups are compared with 2 -way ANOVA $(P<0.05) .{ }^{*} P<0.05$, unpaired $t$ test versus control sham mice.

signaling have the same downstream metabolic consequences. In contrast, in the VMH, leptin negatively regulates FI, promoting weight loss, whereas insulin suppresses EE, leading to weight gain. Ptpn1 deletion in VMH SF-1 neurons causes increased leptin sensitivity and reduced FI, but a concomitant, greater increase in body weight and adiposity in female mice on HFD than in controls. The increased weight gain in this model is likely explained by increased INSR signaling in these neurons, which reduces sympathetic tone and spontaneous locomotor activity. Over time, the effects of SF-1 Ptpn1 deletion on insulin action appear to overcome the effects on leptin action, resulting in obesity. These data provide insight into the integration of different signaling pathways in specific types of hypothalamic neurons involved in the regulation of energy balance.

\section{Methods}

Animal care. Mice were housed at RT $\left(22-24^{\circ} \mathrm{C}\right)$ with a 14 -hour light/10-hour dark cycle (lights on at $6 \mathrm{am}$ ) and were fed standard chow (Teklad Rodent Diet no. 5053; 5\% kcal from fat; $3.1 \mathrm{kcal} / \mathrm{g}$ ) or a HFD (Research Diet D12492i; 60\% kcal from fat; 5.24 kcal/g). Food and water were provided ad libitum.

Generation of mice with SF-1-specific Ptpn1 deletion. To delete Ptpn1, the gene encoding PTP1B, specifically in SF-1 neurons, mice expressing Cre recombinase under control of the $S f 1$ promoter (12) ( a gift from Bradford Lowell, Division of Endocrinology, Diabetes and Metabolism, Beth Israel Deaconess Medical Center-Harvard Medical School) were crossed with mice bearing a floxed Ptpn1 allele (Ptpn $\left.1^{f l o x / f l o x}\right)$, which we generated previously (23), yielding Sf1-Cre Ptpn $1^{f l o x /+}$ mice. These mice were subsequently crossed to

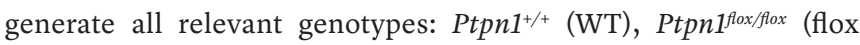
control), Sf1-Cre Ptpn1 ${ }^{++}$(Cre control), and Sf1-Cre Ptpn1 $1^{f l o x / f l o x}$ (Sf1Ptpn1-/). Two initial cohorts were obtained in this manner and were placed on chow or HFD for phenotypic characterization. Control genotypes (WT, Flox control, Cre control) were indistinguishable in body weight, adiposity, and FI. Consequently, subsequent litters were generated via a simplified breeding scheme in which Sf1-Cre
Ptpn $1^{f l o x / f l o x}$ males were bred to Ptpn $1^{f f o x / f l o x}$ females. Body weight was monitored weekly in all animals, and FI was measured on individually housed animals when indicated.

Body composition. Body fat was assessed by MRI, using an EchoMRI (Echo Medical System). Percentage of body fat was expressed as grams of fat/body weight (in grams).

Blood tests. Fed or fasted (12-hour overnight) mice were bled between 8 and $10 \mathrm{am}$. Serum was separated from blood by centrifugation and assayed for insulin and leptin (Crystal Chem). Blood glucose was assessed with a One Touch Ultra Glucometer (Lifescan Inc). T4 and T3 (Coat-A-Count, Siemens), and estradiol and corticosterone levels were quantified by radioimmunoassay (MP Biochemicals Immunochem Double Ab I 125 RIA). Serum testosterone (Testosterone ELISA; MP Biochemicals) and norepinephrine levels (ALPCO Diagnostics, Norepinephrine Research ELISA) were determined by ELISA. All assays were performed per the manufacturer's instructions.

Corticosterone stress test. Corticosterone levels were measured at baseline and in response to the stress of temporary restraint. Beginning 2 weeks prior to the experiment, animals were handled regularly each day for a 10- to 15-minute period between 8 am and 10 am in order to acclimate them to handling. At 8 am on the day of the experiment, 50 to $75 \mu \mathrm{l}$ of serum was collected $(t=0)$ to assess basal corticosterone. Immediately thereafter, each animal was placed in a restraint tube (Medium Mouse Holder; Kent Scientific Corp.). Serum was collected after 30 minutes of restraint ( $t=30$ minutes), after which mice were removed from restraint and returned to their home cage. Serum was collected again at 90 minutes ( $t=90$ minutes) to assess recovery from stress.

Intraventromedial hypothalamus injection. Mice were anesthetized with ketamine (100 mg/kg i.p.) and xylazine (10 mg/kg i.p.). Scalp fur was shaved and prepared using povidone-iodine USP (Medline) and alcohol $70 \%$ prep pads (Dynarex), and mice were placed into a stereotaxic device (Kopf Instruments) with lambda and bregma oriented at the same vertical coordinate. A small midline incision was made over the dorsal scalp to provide access to the cranium, and the cranial surface was cleaned by swabbing with acetone and the allowed to dry. Coordinates for cannula placement to target the 
$\mathrm{VMH}$ were $1.4 \mathrm{~mm}$ posterior to bregma $(\mathrm{AP}=-1.4)$ and $0.25 \mathrm{~mm}$ lateral to the midline $(\mathrm{L}= \pm 0.25)$. A skull window was outlined with a fine drill, and a 26-gauge stainless steel cannula ( $8 \mathrm{~mm}$ below pedestal; PlasticsOne) was lowered until it reached the meninx to establish "0". The cannula was subsequently placed at $-5.3 \mathrm{~mm}$ for the dorsoventral coordinates (43).

On experimental days, $2 \mu \mathrm{l}$ of vehicle (aCSF; Harvard Apparatus) or $4 \mu \mathrm{U} / \mu \mathrm{l}$ of insulin (Humulin R; Eli Lilly) were infused through the cannula over a 30 -second period (43). The guide cannula was kept in place for an additional 10 minutes to allow the drug to diffuse away from the cannula tip. Mice were sacrificed at the end of the injection, and brains were dissected and prepared for immunohistochemistry.

Immunohistochemistry and histology. Mice were deeply anesthetized and perfused with $10 \%$ formalin, and their brains were removed and fixed in $4 \%$ paraformaldehyde in PBS (Sigma-Aldrich) overnight at $4^{\circ} \mathrm{C}$. Whole brains were cryoprotected in $25 \%$ sucrose in $0.1 \mathrm{M}$ PBS at $4^{\circ} \mathrm{C}$ until sectioning. Serial coronal 20 to $30 \mu \mathrm{m}$-thick sections through the rostrocaudal extent of the hypothalamus were cut on a sliding freezing microtome (Leica SM2010R; Leica Microsystems); every fourth section was collected, placed in cryoprotectant solution $(30 \% \mathrm{w} / \mathrm{v}$ ethylene glycol, $20 \% \mathrm{w} / \mathrm{v}$ glycerol, $50 \% \mathrm{w} / \mathrm{v}$ formaldehyde), and stored at $-20^{\circ} \mathrm{C}$ until processed for immunohistochemistry or immunofluorescence (see below). Sections containing the VMH (bregma -1.20 to $-2.22 \mathrm{~mm}$ ) were analyzed by immunohistochemistry, as described $(57,58)$. Immunostaining was performed using rabbit polyclonal pAKT (S473 D9E; 1:50; Cell Signaling Technology) and pFOXO1 (\#28280, 1:50; Cell Signaling Technology) antibodies, based on previous publications $(52,59)$. Primary antibodies were detected by using anti-rabbit IgG secondary antibodies, VECTASTAIN Elite plus ABC kits, and NovaRed kits (Vector Laboratories Inc.). Slides were examined microscopically, photographed using a model of Scanscope 6 slides (Aperio CS) imaging, and analyzed by Colapix software v3.4.3 (Tribun).

Immunofluorescence staining and imaging. lsl-tdTomato is a loxPflanked STOP cassette (lsl) that prevents transcription of the red fluorescent protein tdTomato. The lsl-tdTomato reporter mice from The

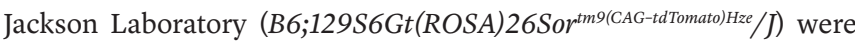
bred with Sf1-Cre Ptpn $1^{f l o x f f l o x}$ (Sf1-Ptpn1-/) to visualize SF-1-positive neurons by immunofluorescence. To minimize variability, matched sections from experimental and control animals were processed simultaneously during each immunostaining procedure. Briefly, free-floating sections were washed 6 times for 10 minutes each time in $0.1 \mathrm{M}$ PBS, pH 7.4, and treated 4 times for 15 minutes each time with $1 \mathrm{mg} / \mathrm{ml}$ $\mathrm{NaBH} 4$ in 0.1 M PBS to reduce background autofluorescence. Sections were blocked for 1 hour in $20 \%$ donkey serum (in $0.2 \%$ Triton $\mathrm{X}-100,0.1 \%$ BSA in $0.1 \mathrm{M}$ PBS) and then incubated for 24 hours at RT with goat anti-PTP1B antibodies (1:100) (AF3954; R\&D Systems Inc.) diluted in $2 \%$ donkey serum (in $0.2 \%$ Triton $\mathrm{X}-100,0.1 \%$ BSA in $0.1 \mathrm{M}$ $\mathrm{PBS})$. Sections were rinsed 3 times for 10 minutes each time in $0.1 \mathrm{M}$ PBS and incubated for 2 hours with Alexa Fluor 488 donkey anti-goat IgG (1/250, A11055. Life Technologies) diluted in $2 \%$ donkey serum (Vector Laboratories, Inc.) Sections were subsequently rinsed 3 times for 10 minutes each time in $0.1 \mathrm{M}$ PBS, then adhered onto Fisherbrand SuperFrost/Plus microscope slides (12-550-15; Fisher Scientific), placed under coverslip (12-545-E; Fisherbrand Microscope Cover Glass) using Vectashield Hard Set mounting medium (H-1400; Vector Labs Inc.), and dried overnight in the dark.
Images were obtained using a Zeiss LSM 510 Meta Confocal Microscope at low and high magnifications $(\times 10, \times 20$, and $\times 63)$. Single-labeled PTP1B-positive neurons (laser excitation lines, $488 \mathrm{~nm}$ ) were scanned from the mediobasal hypothalamus ( $\mathrm{MBH}), \mathrm{VMH}$, and LH (as a positive control region) from $\mathrm{Ptpn1}^{-/-}$and WT littermate control mice. Double-labeled PTP1B-tdTomato neurons in the $\mathrm{MBH}$, VMH, and LH of Sf1-Cre Ptpn1 $1^{f t o x / f l o x} l s l$-tdTomato mice and SF1-Cre Ptpn $1^{f o x /+} l s l$-tdTomato mice were scanned at laser excitation lines 488 $\mathrm{nm}$ (for PTP1B) and $555 \mathrm{~nm}$ (for tdTomato).

$E E$. Metabolic rates were measured by indirect calorimetry with the 16-chamber open-circuit Comprehensive Laboratory Animal Monitoring System (CLAMs (Oxymax; Columbus Instruments), following a previously published protocol (57). To analyze data collected by indirect calorimetry, we followed recommendations established previously (54). Oxygen consumption $\left(\mathrm{VO}_{2}\right), \mathrm{EE}$, and body composition by MRI were assessed in HFD-fed female mice (control, $n=5$; Sf1-Ptpn1 ${ }^{-1-}, n=8$ ) at the beginning of body weight divergence (16-17 weeks of age for this cohort; control BW $=25.3 \pm 1.5 \mathrm{~g}$ vs. Sfl-Ptpn1 $\left.1^{-/-} \mathrm{BW}=29.8 \pm 1.5 \mathrm{~g}, \mathrm{P}=0.04\right) . \mathrm{VO}_{2}$ and $\mathrm{EE}$ were normalized to lean body mass, and analysis of covariance (ANCOVA) was performed (54). $\mathrm{VO}_{2}$ was also assessed in mice on HFD given either i.p. saline or leptin ( $5 \mathrm{mg} / \mathrm{kg}$, 3 times every 12 hours).

Voluntary activity measurements. Female mice were placed in cages outfitted with running wheels connected to probes (Lafayette Instrument Co.) under a 14-hour dark/10-hour light cycle with free access to the wheel, food, and water. Running distance was recorded using Activity Wheel Monitoring Software (AWM; version 11.12) and analyzed with ClockLab/MatLab (version 7.10) over a period of 10 days. The total running distance was quantified, and an actogram for each mouse was generated to observe the pattern of voluntary activity.

Cold exposure and CBT. CBT was determined using a rectal probe (Physitemp Instruments Thermalert Model TH-8). Readings were obtained twice daily ( 8 am and $8 \mathrm{pm}$ ) in mice maintained at RT for 3 consecutive days, and a mean CBT for each time point was established for each animal. Mice ( 27 weeks old) were subjected to a 72-hour cold challenge $\left(4^{\circ} \mathrm{C}\right)$. The $\mathrm{CBT}$ of each animal was monitored every hour over the first 6 hours and again at 12, 24, 36, and 72 hours. Body weight and FI for each animal were recorded at the beginning and end of the experiment.

i.p. glucose tolerance test. Mice were fasted for 6 hours prior to the start of each experiment. After an initial fasted blood sample $(t=0)$ was collected via the tail vein, animals were given a bolus of glucose (1 g/kg body weight i.p., 20\% dextrose; Baxter). Blood glucose readings were taken at $15,30,60,120$, and 180 minutes after injection, using the One-Touch Ultra Glucometer (LifeScan).

Fertility. To assess potential reproductive effects of Ptpn1 deletion in SF-1-expressing cells of the gonads, we monitored fertility by breeding $S f 1$-Ptpn $1^{-/-}$female mice with Ptpn $1^{f l o x f f l o x}$ male mice and Sf1$P t p n 1^{-/-}$male mice with Ptpn $1^{f l o x / f f o x}$ female mice. Gestation time, number of pups per litter, genotype distribution, and serum testosterone and estradiol- $17 \beta$ levels were determined.

Leptin sensitivity test. To determine baseline values, mice were injected with saline twice a day for 3 consecutive days, and FI and body weight were recorded. Then, leptin $(1.5 \mathrm{mg} / \mathrm{kg}$ i.p., National Hormone and Peptide Program [NHPP]) was administered 2 times per day for 3 more days, and FI and body weight were monitored daily.

Real-time quantitative PCR. Total RNA was extracted from BAT harvested from animals at RT and after 72 hours of cold exposure, 
using TRIzol, BCP, and isopropanol; levels were quantified with the NanoDrop-ND1000 (Thermo Scientific). cDNAs were generated by using the Advantage RT-for-PCR kit (Clontech Laboratories Inc.), and SYBR Green PCR master mixes (Applied Biosystems; Life Technologies) were used to quantify peroxisome proliferator-activated receptor gamma coactivator 1 alpha (Pgc1 $\alpha$ ), deiodinase2 (Dio2), Ucp1, Ucp2, Ucp3, leptin (Obrb), and 18S RNA levels, as described (60).

ARC, DMH/VMH, and PVN were microdissected according to coordinates from the mouse brain atlas (61). Total mRNA was extracted from each nucleus, cDNA was generated, and the following primers were used for quantitative real-time PCR, as described previously: ERa: forward 5'-CCTCCCGCCTTCTACAGGT-3', reverse: 5'-CACACGGCACAGTAGCGAG-3' (NCBI GeneID: 13982); ERß: forward: 5'-CTGTGATGAACTACAGTGTTCCC-3', reverse: 5'-CACATTTGGGCTTGCAGTCTG-3' (NCBI GeneID: 13983); and 18S: forward 5'-AGTCCCTGCCCTTTGTACACA-3', reverse: 5'-GATCCGAGGGCCTCACTAAAC-3' (62). ER $\alpha$ and ER $\beta$ levels were quantified by using SYBR Green PCR Master Mix according to the manufacturer's instructions (Applied Biosystems; Life Technologies). Q-RT-PCR was performed by using a 7900 HT PCR machine (Applied Biosystems; Life Technologies). Gene expression levels were normalized to 18S RNA levels and data analyzed with sds 2.3 software (Applied Biosystems; Life Technologies). For each sample, the gene to $18 \mathrm{~S}$ ratio was calculated based on an arbitrary value of copies determined by the standard curve for each gene and normalized to the control group, as described (57).

Ovariectomy and estrogen pellet implantation. Twelve-week-old male Sf1-Ptpn $1^{-/-}$mice $(n=7)$ and their Ptpn $1^{f l o x / f l o x}(n=5)$ controls, both groups on HFD, were anesthetized with isoflurane. Pellets containing estradiol-17 $\beta(2 \mu \mathrm{g} / \mathrm{d} /$ mouse with $90 \%$ releasing capacity per the manufacturer; Innovative Research of America) or placebo $(n=7$ and 4 , respectively) were implanted s.c. Body weight and FI were recorded over the subsequent 55 days, and feed efficiency was calculated.
Twelve-week-old female Sf1-Ptpn $1^{-/-}$mice $(n=7)$ and Ptpn $1^{f l o x / f l o x}$ $(n=5)$ controls were anesthetized with ketamine $(100 \mathrm{mg} / \mathrm{kg})$ and xylazine $(10 \mathrm{mg} / \mathrm{kg})$ and subjected to bilateral ovariectomy $(n=7)$ or sham surgery $(n=5)$, respectively. Postoperatively, mice were maintained on HFD, and body weight and FI were recorded for 66 days, at which time serum estradiol-17 $\beta$ levels were assessed.

Statistics. Data are presented as the mean \pm SEM. Differences between 2 groups were determined using 2-tailed unpaired or paired Student's $t$ tests, as appropriate. Comparisons of multiple groups were performed using ANOVA, followed by a post-hoc analysis by Fisher's LSD test. ANCOVA test was used to compare linear regressions of feed efficiency and EE data (54). For all statistical analyses, a $P$ value of less than 0.05 was considered significant.

Study approval. All procedures involving mice were approved by the Institutional Animal Care and Use Committee at Beth Israel Deaconess Medical Center.

\section{Acknowledgments}

This work was supported by NIH R01 DK060838 (to B.B. Kahn and B.G. Neel), NIH P30 DK57521 (to B.B. Kahn), NIH R37 CA49152 (to B.G. Neel), and a grant from the JPB Foundation (to B.B. Kahn). B.G. Neel is a Canada Research Chair, Tier 1, and work in his lab is partially supported by the Princess Margaret Cancer Foundation and the Ontario Ministry of Health and Long Term Care. K.J. Catalano was supported by NIH Ruth L. Kirschstein National Research Service Award grant DK821642. J. Lee was supported by NIH 5T32DK07516-29 (PI: B. Kahn).

Address correspondence to: Barbara B. Kahn, 330 Brookline Avenue, Boston, Massachusetts 02215, USA. Phone: 617.735.3324; E-mail: bkahn@bidmc.harvard.edu. Or to: Benjamin G. Neel, 101 College Street, TMDT 8-701, Toronto, Ontario, Canada. M5G1L7. Phone: 416.581.7710; E-mail: bneel@uhnresearch.ca.
1. Finkelstein EA, Trogdon JG, Cohen JW, Dietz W. Annual medical spending attributable to obesity: payer-and service-specific estimates. Health Aff (Millwood). 2009;28(5):w822-w831.

2. Mercer JG, et al. Localization of leptin receptor mRNA and the long form splice variant (Ob- $\mathrm{Rb})$ in mouse hypothalamus and adjacent brain regions by in situ hybridization. FEBS Lett. 1996;387(2-3):113-116.

3. Cohen P, et al. Selective deletion of leptin receptor in neurons leads to obesity. JClin Invest. 2001;108(8):1113-1121.

4. Balthasar $\mathrm{N}$, et al. Leptin receptor signaling in POMC neurons is required for normal body weight homeostasis. Neuron. 2004;42(6):983-991.

5. Kim KW, et al. SF-1 in the ventral medial hypothalamic nucleus: a key regulator of homeostasis. Mol Cell Endocrinol. 2011;336(1-2):219-223.

6. Bergen HT, Mizuno TM, Taylor J, Mobbs CV. Hyperphagia and weight gain after gold-thioglucose: relation to hypothalamic neuropeptide $\mathrm{Y}$ and proopiomelanocortin. Endocrinology. 1998;139(11):4483-4488.

7. Schwartz MW, et al. Central nervous system control of food intake. Nature. 2000;404(6778):661-671.

8. Jacob RJ, et al. The effect of leptin is enhanced by microinjection into the ventromedial hypothalamus. Diabetes. 1997;46(1):150-152.

9. Satoh $\mathrm{N}$, et al. The arcuate nucleus as a primary site of satiety effect of leptin in rats. Neurosci Lett. 1997;224(3):149-152.

10. Satoh N, et al. Sympathetic activation of leptin via the ventromedial hypothalamus: leptin-induced increase in catecholamine secretion. Diabetes. 1999;48(9):1787-1793.

11. Bingham NC, et al. Selective loss of leptin receptors in the ventromedial hypothalamic nucleus results in increased adiposity and a metabolic syndrome. Endocrinology. 2008;149(5):2138-2148.

12. Dhillon $\mathrm{H}$, et al. Leptin directly activates SF1 neurons in the $\mathrm{VMH}$, and this action by leptin is required for normal body-weight homeostasis. Neuron. 2006;49(2):191-203.

13. Frangioni JV, et al. The nontransmembrane tyrosine phosphatase PTP-1B localizes to the endoplasmic reticulum via its 35 amino acid C-terminal sequence. Cell. 1992;68(3):545-560.

14. Woodford-Thomas TA, Rhodes JD, Dixon JE. Expression of a protein tyrosine phosphatase in normal and v-src-transformed mouse $3 \mathrm{~T} 3$ fibroblasts. J Cell Biol. 1992;117(2):401-414.

15. Ahmad F, et al. Alterations in skeletal muscle protein-tyrosine phosphatase activity and expression in insulin-resistant human obesity and diabetes. J Clin Invest. 1997;100(2):449-458.

16. Ahmad F, et al. Improved sensitivity to insulin in obese subjects following weight loss is accompanied by reduced protein-tyrosine phosphatases in adipose tissue. Metabolism. 1997;46(10):1140-1145.

17. Ahmad F, Goldstein BJ. Alterations in specific protein-tyrosine phosphatases accompany insulin resistance of streptozotocin diabetes. Am J Physiol. 1995;268(5 pt 1):E932-E940.

18. Ahmad F, Goldstein BJ. Increased abundance of specific skeletal muscle protein-tyrosine phosphatases in a genetic model of insulin-resistant obesity and diabetes mellitus. Metabolism. 1995;44(9):1175-1184.

19. Goldstein BJ. Protein-tyrosine phosphatase 1B (PTP1B): a novel therapeutic target for type 2 diabetes mellitus, obesity and related states of insulin resistance. Curr Drug Targets Immune Endocr Metabol Disord. 2001;1(3):265-275.

20. Kusari J, et al. Skeletal muscle protein tyrosine phosphatase activity and tyrosine phosphatase $1 \mathrm{~B}$ protein content are associated with insulin action and resistance. J Clin Invest. 1994;93(3):1156-1162. 
21. Zabolotny JM, et al. PTP1B regulates leptin signal transduction in vivo. Dev Cell. 2002;2(4):489-495.

22. Cheng A, et al. Attenuation of leptin action and regulation of obesity by protein tyrosine phosphatase 1B. Dev Cell. 2002;2(4):497-503.

23. Bence KK, et al. Neuronal PTP1B regulates body weight, adiposity and leptin action. Nat Med. 2006;12(8):917-924.

24. Morris DL, Rui L. Recent advances in understanding leptin signaling and leptin resistance. Am J Physiol Endocrinol Metab. 2009;297(6):E1247-E1259.

25. Banno R, et al. PTP1B and SHP2 in POMC neurons reciprocally regulate energy balance in mice. J Clin Invest. 2010;120(3):720-734.

26. Dadke S, Chernoff J. Interaction of protein tyrosine phosphatase (PTP) $1 \mathrm{~B}$ with its substrates is influenced by two distinct binding domains. Biochem J. 2002;364(pt 2):377-383.

27. Elchebly M, et al. Increased insulin sensitivity and obesity resistance in mice lacking the protein tyrosine phosphatase-1B gene. Science. 1999;283(5407):1544-1548.

28. Salmeen A, et al. Molecular basis for the dephosphorylation of the activation segment of the insulin receptor by protein tyrosine phosphatase 1B. Mol Cell. 2000;6(6):1401-1412.

29. Klaman LD, et al. Increased energy expenditure, decreased adiposity, and tissue-specific insulin sensitivity in protein-tyrosine phosphatase 1B-deficient mice. Mol Cell Biol. 2000;20(15):5479-5489.

30. Delibegovic M, et al. Improved glucose homeostasis in mice with muscle-specific deletion of protein-tyrosine phosphatase 1B. Mol Cell Biol. 2007;27(21):7727-7734.

31. Delibegovic M, et al. Liver-specific deletion of protein-tyrosine phosphatase 1B (PTP1B) improves metabolic syndrome and attenuates diet-induced endoplasmic reticulum stress. Diabetes. 2009;58(3):590-599.

32. Zabolotny JM, et al. Transgenic overexpression of protein-tyrosine phosphatase $1 \mathrm{~B}$ in muscle causes insulin resistance, but overexpression with leukocyte antigen-related phosphatase does not additively impair insulin action. J Biol Chem. 2004;279(23):24844-24851.

33. Haj FG, et al. Liver-specific protein-tyrosine phosphatase 1B (PTP1B) re-expression alters glucose homeostasis of PTP1B-/-mice. J Biol Chem. 2005;280(15):15038-15046.

34. Klockener T, et al. High-fat feeding promotes obesity via insulin receptor/PI3K-dependent inhibition of SF-1 VMH neurons. Nat Neurosci. 2011;14(7):911-918.

35. Picardi PK, et al. Reduction of hypothalamic protein tyrosine phosphatase improves insulin and leptin resistance in diet-induced obese rats. Endocrinology. 2008;149(8):3870-3880.

36. Picardi PK, et al. Modulation of hypothalamic PTP1B in the TNF-alpha-induced insulin and leptin resistance. FEBS Lett. 2010;584(14):3179-3184.

37. Ramadori G, et al. SIRT1 deacetylase in SF1 neurons protects against metabolic imbalance. Cell Metab. 2011;14(3):301-312.

38. Xu Y, et al. Distinct hypothalamic neurons mediate estrogenic effects on energy homeostasis and reproduction. Cell Metab. 2011;14(4):453-465.

39. Zhang R, et al. Selective inactivation of Socs 3 in SF1 neurons improves glucose homeostasis without affecting body weight. Endocrinology. 2008;149(11):5654-5661.

40. Zhang Y, Scarpace PJ. The role of leptin in leptin resistance and obesity. Physiol Behav. 2006;88(3):249-256.

41. Cao J, Patisaul HB. Sexually dimorphic expression of hypothalamic estrogen receptors alpha and beta and Kiss1 in neonatal male and female rats. J Comp Neurol. 2011;519(15):2954-2977.

42. Yi CX, Scherer T, Tschop MH. Cajal revisited: does the VMH make us fat? Nat Neurosci. 2011;14(7):806-808.

43. Brown LM, Clegg DJ, Benoit SC, Woods SC. Intraventricular insulin and leptin reduce food intake and body weight in C57BL/6J mice. Physiol Behav. 2006;89(5):687-691.

44. Foster LA, Ames NK, Emery RS. Food intake and serum insulin responses to intraventricular infusions of insulin and IGF-I. Physiol Behav. 1991;50(4):745-749.

45. Ikeda $\mathrm{H}$, et al. Intraventricular insulin reduces food intake and body weight of lean but not obese Zucker rats. Appetite. 1986;7(4):381-386.

46. McGowan MK, Andrews KM, Kelly J, Grossman SP. Effects of chronic intrahypothalamic infusion of insulin on food intake and diurnal meal patterning in the rat. Behav Neurosci. 1990;104(2):373-385.

47. Woods SC, Lotter EC, Vogel KW, Porte D Jr. A dual-purpose device for protecting brain implants of baboons. J Neurosci Methods. 1979;1(4):319-322.

48. Elmquist JK, et al. Leptin activates neurons in ventrobasal hypothalamus and brainstem. Endocrinology. 1997;138(2):839-842.

49. Satoh N, et al. Pathophysiological significance of the obese gene product, leptin, in ventromedial hypothalamus (VMH)-lesioned rats: evidence for loss of its satiety effect in VMH-lesioned rats. Endocrinology. 1997;138(3):947-954.

50. Sakaguchi T, Bray GA. Intrahypothalamic injection of insulin decreases firing rate of sympathetic nerves. Proc Natl Acad Sci U S A. 1987;84(7):2012-2014.

51. Tsou RC, Zimmer DJ, De Jonghe BC, Bence KK. Deficiency of PTP1B in leptin receptorexpressing neurons leads to decreased body weight and adiposity in mice. Endocrinology 2012;153(9):4227-4237.

52. Kim KW, et al. FOXO1 in the ventromedial hypothalamus regulates energy balance. J Clin Invest. 2012;122(7):2578-2589.

53. Xu Y, et al. PI3K signaling in the ventromedial hypothalamic nucleus is required for normal energy homeostasis. Cell Metab. 2010;12(1):88-95.

54 . Tschop MH, et al. A guide to analysis of mouse energy metabolism. Nat Methods. 2012;9(1):57-63.

55. Elmquist JK, et al. Leptin activates distinct projections from the dorsomedial and ventromedial hypothalamic nuclei. Proc Natl Acad Sci US A. 1998;95(2):741-746.

56. Kim KW, et al. Steroidogenic factor 1 directs programs regulating diet-induced thermogenesis and leptin action in the ventral medial hypothalamic nucleus. Proc Natl Acad Sci US A. 2011;108(26):10673-10678.

57. Chiappini F, Cunha LL, Harris JC, Hollenberg AN. Lack of cAMP-response element-binding protein 1 in the hypothalamus causes obesity. J Biol Chem . 2011;286(10):8094-8105.

58. Sugrue ML, et al. The thyrotropin-releasing hormone gene is regulated by thyroid hormone at the level of transcription in vivo. Endocrinology. 2010;151(2):793-801.

59. Chalhoub N, Zhu G, Zhu X, Baker SJ. Cell type specificity of PI3K signaling in Pdk1- and Pten-deficient brains. Genes Dev. 2009;23(14):1619-1624.

60. Xue B, et al. Neuronal protein tyrosine phosphatase $1 \mathrm{~B}$ deficiency results in inhibition of hypothalamic AMPK and isoform-specific activation of AMPK in peripheral tissues. Mol Cell Biol. 2009;29(16):4563-4573.

61. Franklin K, Paxinos G. The Mouse Brain In Stereotaxic Coordinates. Waltham, Massachusetts, USA: Academic Press; 2007.

62. Benoit SC, et al. Palmitic acid mediates hypothalamic insulin resistance by altering PKC- $\theta$ subcellular localization in rodents. JClin Invest. 2009;119(9):2577-2589. 